\title{
Diacronie
}

Studi di Storia Contemporanea

$N^{\circ} 25,1 \mid 2016$

"Se creare è definire"

\section{Bo Lidegaard, Il popolo che disse no}

Jacopo Bassi

\section{(2) OpenEdition \\ Journals}

\section{Edizione digitale}

URL: http://journals.openedition.org/diacronie/3890

DOI: 10.4000/diacronie.3890

ISSN: 2038-0925

\section{Editore}

Association culturelle Diacronie

Notizia bibliografica digitale

Jacopo Bassi, « Bo Lidegaard, II popolo che disse no », Diacronie [Online], № 25, 1 | 2016, documento 12 , Messo online il 29 mars 2016, consultato il 24 septembre 2020. URL : http://journals.openedition.org/ diacronie/3890; DOI : https://doi.org/10.4000/diacronie.3890 


\section{Diacronie}

\section{RECENSIONE:}

\section{Bo LIDEGAARD, Il popolo che disse no, Milano, Garzanti, 2014, 455 pp.}

a cura di Jacopo BASSI *

Lo storico Bo Lidegaard ${ }^{1}$ direttore del quotidiano "Politiken» e con una carriera diplomatica alle spalle è il fratello di Martin Lidegaard, membro del Partito SocialLiberale Danese e attuale Ministro degli Esteri della Danimarca. Nel suo profilo biografico si intravedono alcune delle chiavi di lettura necessarie per comprendere il suo Il popolo che disse no, un'interessante narrazione della fuga degli ebrei danesi dal paese durante l'occupazione nazista.

Una prima osservazione riguarda il titolo originale dell'opera - tradotto in modo non fedele ${ }^{2}$ - Landsmænd, che già delinea una delle tesi del libro. Gli ebrei danesi furono sempre considerati come compatrioti dai danesi di religione cristiana; proprio a partire da questo fondamentale presupposto, Lidegaard lega la protezione nei confronti degli ebrei danesi alla difesa della libertà politica danese e all'esistenza stessa del presupposto di Stato di diritto. L'autore afferma sin dalle prime pagine come, ancor prima dell'invasione nazista, si sia creata una forte coesione fra le forze democratiche danesi volta a creare una barriera difensiva - almeno ideologica, dal momento che non era possibile pensare a una difesa militare - di fronte al minaccioso espansionismo tedesco. Proprio per questa ragione, ossia la non appartenenza al campo democratico,

\footnotetext{
${ }^{1}$ Tra le sue pubblicazioni, incentrate sullo studio della diplomazia danese: LIDEGAARD, Bo, $I$ Kongens navn : Henrik Kauffmann i dansk diplomati, 1919-1958, København : Samleren, 1996; ID., Den højeste pris : Povl Bang-Jensen af FN 1955-59, København, Samleren, 1998; ID., Kampen om Danmark 1933-1945, København, Gyldendal, 2005; ID., H.C. Hansens liv og tid : en historie fortalt $i$ billeder, 1906-1960, København, Gyldendal, 2006; ID., A short history of Denmark in the 2oth century, København : Gyldendal, 2009.

${ }^{2}$ Il titolo richiama, inevitabilmente, la pubblicistica che si richiama alle operazioni di salvataggio degli ebrei, frequentemente basati sull'esaltazione della figura dell'uomo solo. Tra gli esempi degli ultimi anni, che non a caso si rifanno al caso bulgaro e, indirettamente, all'atteggiamento italiano verso gli ebrei: NISSIM, Gabriele, L'uomo che fermò Hitler. La storia di Dimită Pešev che salvò gli ebrei di una nazione intera, Milano, Mondadori, 1998; DEAGLIO, Enrico, La banalità del bene: storia di Giorgio Perlasca, Milano, Feltrinelli, 1991. In questo caso il focus è spostato su un intero popolo.
} 
comunisti e nazisti furono stigmatizzati come "non danesi" ed esclusi dalla comunità (politica e nazionale).

Lidegaard, nelle prime pagine, si sofferma sulla questione della difesa militare e della mancata definizione dei confini fra Germania e Danimarca nello Jutland³, per poi concentrarsi sul problema storiografico della politica intrapresa dal governo danese nei confronti della Germania nazista, definita come "politica del negoziato" dai difensori di questo atteggiamento e "politica di collaborazione" dai suoi detrattori, sostenitori della resistenza armata. Lo schema che portò alla realizzazione di quella che la Germania definì "occupazione pacifica" permise alla Danimarca di perdere sovranità solo in materia di politica estera, lasciando al governo di Copenaghen l'amministrazione degli affari interni.

È intorno alla difesa dell'autonomia politica - sostiene Lidegaard facendo riferimento ad una storiografia consolidata - che la Danimarca trovò la capacità di restare coesa superando gli anni della guerra: la politica di deportazione degli ebrei danesi non sarebbe stata accettata e avrebbe aumentato l'esiguo numero di coloro che avevano deciso di imbracciare le armi contro l'occupazione nazista.

Gli stessi nazisti fecero tutto il possibile perché l'occupazione non generasse un'opposizione tra la popolazione danese, cosa che avvenne fino alla promulgazione della legge marziale da parte tedesca.

Personaggio cardine della politica tedesca fu Werner Best, membro delle S.S. e plenipotenziario del Reich nel paese, il cui comportamento oscillò sempre fra la ricerca del compromesso con i politici danesi e la necessità di attuare le direttive naziste. Molto spesso Best preferì attribuire le responsabilità delle scelte che avrebbero potuto suscitare malcontento negli ambienti politici danesi agli ordini provenienti da Berlino o, addirittura, facendo ricadere la colpa delle decisioni prese - come nel caso della scelta di dare luogo al rastrellamento di ebrei - alla Wehrmacht e al suo responsabile in Danimarca, Hermann von Hanneken.

Con la promulgazione della legge marziale - avvenuta il 29 agosto 1943 - fu infatti molto difficoltoso trovare una soluzione per salvaguardare l'autonomia di governo danese; il governo precedentemente in carica si era dimesso e tutti i politici legalmente eletti si rifiutavano di assumere un incarico di governo perché ciò avrebbe significato collaborare attivamente con l'occupante nazista. Di fatto, la crisi di ottobre rappresentò la fine della politica di cooperazione ${ }^{4}$.

\footnotetext{
3 LIDEGAARD, Bo, Il popolo che disse no, Milano, Garzanti, 2014, pp. 27 et seq.

4 KIRCHHOFF, Hans, Augustoprøret 1943. Samarbejdspolitikkens fald: forudsætninger og forløb : en studie i kollaboration og modstand, København, Gyldendal, 1979.
} 
Con le dimissioni del governo, evidenzia la storica dell'olocausto Leni Yahil, si verificò un fenomeno consequenziale: «[...] con l'annullamento delle istituzioni democratiche della Danimarca, in realtà, anche la protezione degli ebrei fu revocata come parte civile della società legale» 5 .

Si creò così un consiglio informale di politici danesi, definito Consiglio dei ministri permanenti e presieduto da Niels Svenningsen, che assunse il compito di mantenere le relazioni con i vertici nazisti in Danimarca. Questi uomini erano Werner Best, Paul Kanstein e Georg Ferdinand Duckwitz; gli ultimi due si dimostrarono due fermi oppositori della linea hitleriana e, anche se non è possibile affermare che lavorarono contro le politiche attuate da Berlino, certamente è possibile dire che agevolarono la fuga degli ebrei danesi verso la Svezia.

La comunità ebraica in Germania si era costituita attraverso tre differenti ondate migratorie: la prima, risalente al XVII secolo, i cui componenti erano ormai totalmente inseriti nella società danese; una seconda era composta da quelli che venivano definiti "ebrei russi", giunti in Danimarca agli inizi del XIX secolo dai paesi dell'Europa orientale; infine, vi erano coloro che erano immigrati più recentemente, per effetto delle persecuzioni naziste in Austria, Germania e Cecoslovacchia.

Nel complesso, secondo Lidegaard, la comunità ebraica danese era perfettamente integrata (non erano infrequenti, peraltro, i casi ebrei di non praticanti): di fatto risultava preminente, a giudizio dell'autore, il loro sentirsi danesi rispetto alla loro fede religiosa ${ }^{6}$.

La consapevolezza dell'impossibilità di sradicare la democrazia e lo Stato di diritto dalla Danimarca, a parere dell'autore, era la miglior garanzia per gli ebrei danesi:

[...] la tenacia degli ebrei danesi nel ritenere che il diritto fosse la loro miglior difesa potrebbe sembrare una pericolosa illusione, un autoinganno, sapendo cosa ne sarebbe seguito, ma non è così semplice. Fu una strategia che contribuì a consolidare la visione della società danese come un tutt'uno di fronte a una minaccia che non riguardava solo coloro che stavano in prima linea, ovvero gli

\footnotetext{
5 Ibidem, p. 79.

${ }^{6}$ La teoria della forte assimilazione della comunità ebraica danese è sostenuta, tra gli altri, da Hans Kirchoff. Andrew Buckser propugna, invece, una teoria radicalmente differente. Fu proprio la capacità di mantenere vive le tradizioni ebraiche a muovere lo spirito solidaristico dei danesi. Buckser si sofferma in particolare sulla corrente religiosa grundtvigiana: molti dei seguaci di questo movimento - e appartenenti alla Danmarks Ungdomssamvirke, l'Unione della Gioventù danese - entrarono nelle file della Resistenza. Per Buckser, fu proprio l'ammirazione per la capacità degli ebrei di mantenere vive le proprie tradizioni e la propria cultura, pur nelle vicissitudini della storia, a farne un simbolo dell'indipendenza danese. BUCKSER, Andrew, «Rescue and Cultural Context During the Olocaust: Grundtvigian Nationalism and the Rescue of the Danish Jews», in Shofar: An Interdisciplinary Journal of Jewish Studies, 19, 2/2001, pp. 125 .
} 
ebrei. La posta in gioco erano i fondamenti stessi di uno stato di diritto e, quindi, tutto ciò per cui i danesi avevano lottato nel corso dei decenni7.

Come ebbe modo di ricordare anche Leni Yahil, gli ebrei, per i danesi, non furono dunque una questione umanitaria, ma innanzitutto politica ${ }^{8}$.

La stessa logica - accettare un controllo parziale piuttosto che soccombere di fronte al controllo di un governo collaborazionista - venne applicata anche nei confronti della stampa: censura e autocensura venivano considerate preferibili rispetto al controllo diretto tedesco.

Il delicato equilibrio che si venne a creare in Danimarca si basava dunque su due paure contrapposte: da una parte i politici danesi temevano di perdere la sovranità interna, dall'altra i nazisti non volevano che l'occupazione pacifica si trasformasse in un'occupazione militare tout court, che avrebbe richiesto molte più truppe per controllare il territorio.

Fu proprio a partire dalla necessità di mantenere lo status quo che le ambiguità e i temporeggiamenti di un personaggio come Best - che con i suoi interlocutori danesi negò sino all'ultimo l'esistenza di un'operazione di rastrellamento degli ebrei danesi poterono consentire a Duckwitz di diffondere la notizia.

Questa mossa, assieme alla contemporanea trasmissione della notizia ai vertici politici svedesi, permisero il buon esito dell'operazione.

Ł̀ tuttavia altamente improbabile che Best non fosse al corrente della fuga di notizie: con ogni probabilità aveva accondisceso a questa soluzione preferendo una sorta di "politica di espulsione" come soluzione alternativa da adottare nei confronti degli ebrei danesi9.

La diffusione della notizia attraverso il passaparola permise così di ridurre al minimo il numero degli ebrei che caddero vittima dell'operazione nazista il $1^{\circ}$ ottobre.

La chiave dell'insuccesso dell'operazione, secondo Lidegaard, risiede nel fatto che: «[...] si svolse sotto gli occhi di una società estremamente indignata e protettiva, e fu seguita passo dopo passo dalla stampa svedese, per così dire. Questo è esattamente il

\footnotetext{
7 Ibidem, pp. 78-79.

8 YAHIL, Leni, The Rescue of the Danish Jewry: Test of a Democracy, Philadelphia, Jewish Publication Society of America, 1969.

9 Gunnar Paulson sottolinea l'importanza di quanto avvenuto in Italia l'8 settembre: questi eventi non potevano che far propendere per un maggior impiego di forze sul fronte italiano, alleggerendo il peso militare sulla Danimarca e rendendo perciò necessario limitare ogni sforzo. PAULSON, Gunnar S., «The 'Bridge over the Øresund': The Historiography on the Expulsion of the Jews from Nazi-Occupied Denmark», in Journal of Contemporary History, 30, 3/1995, pp. 431-464, p. 455.
} 
motivo per cui in questo caso l'apparato nazista fece un buco nell'acqua» ${ }^{10}$. L'autore sottolinea come gli ebrei danesi, in fuga dal paese, trovarono un luogo di rifugio in Svezia, dove l'elettorato era radicalmente contrario all'antisemitismo e al nazismo e lo Stato svedese si prese cura dei rifugiati.

I fuggitivi, infatti, furono costretti a raggiungere le coste svedesi con mezzi di fortuna, approfittando delle imbarcazioni dei pescatori. Lidegaard sottolinea lo sforzo altruistico profuso dalla popolazione danese per metterli in salvo ${ }^{11}$, pur non negando che: «La questione ha tormentato il dibattito sul salvataggio fin da quei drammatici giorni di ottobre, e già all'epoca non mancavano le osservazioni sarcastiche sui guadagni dei pescatori $\left[\ldots . . »^{12}\right.$.

L'autore propone ai lettori una narrazione storica in cui si fondono tre distinte vicende: quella degli ebrei danesi; quella dei personaggi impegnati in ambito politico e diplomatico; la storia politica danese ed europea. L'intento è quello di mostrare la stretta interconnessione fra la macrostoria e le storie personali.

L'evoluzione della vicenda viene seguita - ed è questo il punto di maggior originalità e interesse del libro - attraverso gli stralci dei diari di coloro che vissero questi eventi: i passaggi sono intervallati da precisi e puntuali contestualizzazioni.

Lidegaard sottolinea a più riprese come non vi fosse piena consapevolezza tra $\mathrm{i}$ politici danesi del destino a cui andavano incontro gli ebrei che venivano deportati dai nazisti: questa ricostruzione è discutibile nella misura in cui è l'autore stesso a dare conto dei suicidi verificatisi tra gli ebrei danesi per non cadere in mano tedesca ${ }^{13}$. Riesce difficile credere che i membri della comunità ebraica potessero avere accesso a informazioni che non fossero in mano anche ai vertici della politica danese.

Appare molto debole anche la logica di Lidegaard di fronte della disparità di comportamento adottata dalla classe politica danese nei confronti dei comunisti rispetto agli ebrei. L'autore sottolinea - nella prima parte del volume - come nazisti e comunisti danesi si fossero automaticamente autoesclusi dalla comunità politica (basata sull'ideologia democratica) e avessero posto i presupposti per essere considerati "non danesi"; questo schema interpretativo viene ribadito e rafforzato:

Sebbene l'odio per i tedeschi non facesse che crescere, molti mantenevano anche forti riserve sul fatto che i comunisti assumessero un ruolo di primo piano nella

10 LIDEGAARD, Bo, Il popolo che disse no, cit., pp. 315-316.

${ }^{11}$ La tesi è sostenuta con forza dallo storico danese Hans Kirchhoff. Si veda: KIRCHHOFF, Hans, «A Light in the Darkness of the Holocaust? A Reply to Gunnar S. Paulsson», in Journal of Contemporary History, 30, 3/1995, pp. 465-479.

12 LIDEGAARD, Bo, Il popolo che disse no, cit., p. 389.

13 Ibidem, p. 281. 
lotta armata. Per la stessa ragione, i politici eletti nutrivano ancora un certo scetticismo nei confronti dei militanti armati per la libertà. Quali sarebbero state le loro intenzioni se l'Unione Sovietica avesse vinto la guerra? Anche se i comunisti avevano iniziato a usare slogan nazionali, inneggiando alla sconfitta del fascismo e della liberazione della Danimarca, pochi si fidavano del loro sentimento patriottico e molti temevano che stessero usando la lotta contro i tedeschi come un trampolino di lancio per il loro continuo desiderio di sconvolgimenti sociali e di rivoluzione sul modello sovietico ${ }^{14}$.

Ł̀ sulla scorta di queste considerazioni che deve essere interpretata, secondo Lidegaard, la legislazione anticomunista varata in Danimarca. Infatti, il giornale comunista «Frit Denmark» commentando le deportazioni di comunisti danesi, equiparava la loro situazione a quella minoranza di ebrei danesi caduti vittima del rastrellamento nazista:

La battaglia infuria ed esige le sue vittime: 143 comunisti danesi sono stati deportati al campo di concentramento di Stutthof, vicino a Danzica, fianco a fianco con gli ebrei. E nessuno può sapere chi sarà il prossimo ${ }^{15}$.

Sulle ragioni della salvezza degli ebrei danesi Lidegaard non ha dubbi di sorta ${ }^{16}$ :

Ciò che in definitiva fermò lo sterminio degli ebrei in terra danese fu la manifesta $\mathrm{e}$ radicata opposizione dei danesi a tale disegno. Questo, insieme alla comprensione del cinico compromesso tra i diversi obiettivi strategici che guidavano i vertici nazisti nell'operazione contro la Danimarca e nell'operazione contro gli ebrei danesi apre una prospettiva inquietante: i responsabili si tirarono indietro quando si trovarono a dover scegliere in maniera chiara tra il perseguire i loro interessi generali in Danimarca e il perseguitare gli ebrei danesi ${ }^{17}$.

Quel che appare scricchiolare sotto il peso del confronto con il destino occorso ai comunisti danesi è uno degli assurti fondamentali del libro:

${ }^{14}$ Ibidem, p. 304.

${ }^{15}$ Ibidem, p. 306.

16 Una lettura radicalmente diversa viene fornita da Paulsson; questi benché riconosca il generale comportamento solidale della popolazione danese, attribuisce in larga misura ai vertici nazisti in Danimarca la volontà di far fallire il piano di rastrellamento degli ebrei, consentendone la fuga. PAULSSON, Gunnar, op. cit.

17 LIDEGAARD, Bo, Il popolo che disse no, cit., p. 403. 
[...] il fatto che la società danese si rivoltò [...] contro l'idea stessa alla base della persecuzione dei propri connazionali. Un atteggiamento, questo, ancorato ai precedenti dieci anni di politica antitotalitaria danese: al di fuori di tale contesto politico, l'esodo miracoloso degli ebrei danesi non può essere compreso ${ }^{18}$.

Pertanto, o si sposa la teoria per cui i comunisti danesi erano realmente considerati "non danesi" dai loro compatrioti, oppure riesce difficile poter accettare questa spiegazione alla base della salvezza di gran parte della comunità ebraica danese.

Il libro si propone come una preziosa ricostruzione storica degli anni del secondo conflitto mondiale in Danimarca (questione non particolarmente nota in Italia); il quadro che viene restituito dall' autore è impreziosito dall'utilizzo dei diari come fonti privilegiate, attorno alle quali si sviluppa una narrazione che riesce nell'intento di restituire sia il vissuto dei protagonisti, sia il loro intersecarsi con le vicende politiche e diplomatiche su scala nazionale e internazionale. L'intento di Lidegaard non è quello di rivolgersi ad un pubblico di addetti ai lavori: il merito è di rivolgersi ad un pubblico generalista facendo ricorso a documenti archivistici e al rigore scientifico nella ricostruzione storica. 


\section{* L'autore}

Jacopo Bassi ha conseguito la laurea in Storia contemporanea (Università degli Studi di Bologna). Nel corso dei suoi studi si è occupato di storia della Grecia e dell'Albania in età contemporanea e di storia e istituzioni della Chiesa ortodossa. Ha lavorato per le case editrici Éditions des femmes, Il Mulino e Zanichelli.

URL: < http://www.studistorici.com/2009/02/24/jacopo_bassi/ >

\section{Per citare questo articolo:}

BASSI, Jacopo, «Recensione: Bo LIDEGAARD, II popolo che disse no, Milano, Garzanti, 2014, 455 pp.», Diacronie. Studi di Storia Contemporanea : "Se creare è definire", 29/03/2016,

URL:< http://www.studistorici.com/2016/03/29/bassi_numero_25/ >

Diacronie Studi di Storia Contemporanea $\}$ www.diacronie.it

Risorsa digitale indipendente a carattere storiografico. Uscita trimestrale.

redazione.diacronie@hotmail.it

Comitato di redazione: Jacopo Bassi - Luca Bufarale - Elisa Grandi - Antonio César Moreno Cantano - Deborah Paci - Fausto Pietrancosta - Alessandro Salvador - Matteo Tomasoni - Luca Zuccolo

Diritti: gli articoli di Diacronie. Studi di Storia Contemporanea sono pubblicati sotto licenza Creative Commons 3.0. Possono essere riprodotti e modificati a patto di indicare eventuali modifiche dei contenuti, di riconoscere la paternità dell'opera e di condividerla allo stesso modo. La citazione di estratti è comunque sempre autorizzata, nei limiti previsti dalla legge. 